\title{
Adverse Reactions to Oral Antidiabetic Agents
}

\author{
E. L. HARRIS
}

British Medical fournal, 1971, 3, 29-30

\section{Introduction}

Unfortunately, at present we do not have the facilities which allow adequate monitoring of the whole population or even selected groups to detect possible serious morbidity or mortality as a result of drug administration. The Committee on Safety of Drugs has depended on an "early warning" system based on the collection and assessment of adverse reaction reports and data on drug usage. The main source of information is the voluntary reporting of adverse reactions by doctors, dentists, and pharmaceutical companies. The Committee provides a simple reply-paid "yellow card" report form for this purpose. Each report is analysed by a team of three full-time physicians and the data are then fed into a central computer, which produces a cumulative tabulation for consideration by the Committee at its regular meetings. When necessary, if the reports are incomplete a member of a team of 40 part-time doctors can obtain additional information by visiting the clinicians concerned.

Each year the Committee receives about 3,500 reports of adverse reaction. Nevertheless, there is still a gross underreporting of adverse reactions, judging by the Committee's experience of drugs that have been specially investigated. The importance of the oral antidiabetic drugs and the present controversy over their safety has prompted an analysis of the more important adverse reactions reported to the Committee since May 1964.
Committee on Safety of Drugs, London, EC2A 1PP E. L. Harris, M.B., M.R.C.P., Senior Medical Officer

\section{Background}

Before considering the breakdown of reactions, some knowledge of how often the various drugs are prescribed is needed so that the relative importance of each reaction can be determined (see Table).

Prescriptions for Antidiabetic Drugs issued by General Practitioners in England, Scotland and Wales during 1969

\begin{tabular}{ll|c}
\hline \multicolumn{1}{c|}{ Agent } & No. of Prescriptions \\
\hline Insulin . . &.. & 652,000 \\
Chlorpropamide & 564,000 \\
Tolbutamide $\quad .$. & 324,000 \\
Phenformin &. & 155,000 \\
Metformin &.. & 54,000 \\
Tolazamide &.. & 20,000 \\
Acetohexamide &.. & 16,000 \\
\hline
\end{tabular}

The prescribing trend for 1970 shows a slight fall in the number of insulin and an increase in chlorpropamide prescriptions. Unfortunately, the figures for hospital usage of these drugs are not readily available. Few doctors have difficulty in deciding when a serious reaction to a drug should be reported to the Committee. Nevertheless, "grey" areas do exist-for example, should the occurrence of hypoglycaemia be notified? Most regard this as an extension of the pharmacological action of the drug and consider it too trivial to report. Our register, therefore, gives a gross underestimate of this event, which, incidentally, if unexpected or unusual in any way should be regarded as an adverse reaction and reported. Diabetics, unfortunately, are not immune to other disorders and many of the reports contain details of concurrent treatment. This poses the problem of deciding which drug caused the reaction. About 
one-third of the reports received do not contain enough information about the reaction, dosage, duration of treatment, other drugs, and the outcome of the reaction. Initially this was partly due to the design of the yellow form; however, this has been revised and the quality of reporting has been much improved.

I intend to deal only with the four most commonly used oral antidiabetic agents.

\section{Chlorpropamide}

Sixty-one valid reports of reaction to chlorpropamide were received, the most common being a variety of skin reactions in 25 patients. These include Stevens-Johnson syndrome, exfoliative dermatitis, eczema, photodermatitis, erythema nodosum, purpuric rashes, and papular rashes. One patient developed alopecia areata, which cleared when treatment was changed to insulin and there was one case of generalized pruritus. Most of the skin lesions appeared within 7 to 21 days after beginning treatment, suggesting sensitization, possibly from previous sulphonamide therapy.

The next most common adverse effects were on the blood: aplastic anaemia three, agranulocytosis two, pancytopenia three, leucopenia four, thrombocytopenia five, and haemolytic anaemia two. The onset was either within one month of starting treatment or after a period of six months to seven years.

Liver damage usually accompanied by jaundice occurred in eight cases; these were mainly of the cholestatic type but hepatocellular damage and cirrhosis were also reported. Onset was within two months or after four to eight years. There were five cases of diarrhoea and five of alcohol induced side effects; these will be discussed under drug interactions.

\section{Tolbutamide}

Half the 14 reports on tolbutamide were of rash, including eczema and macular erythema. Here, unlike experience with chlorpropamide, the onset occurred from six months to two years after starting treatment. There were two blood dyscrasias, both case of aplastic anaemia, which came on after four and seven years of therapy. Two cases of severe hypoglycaemia precipitated by phenylbutazone were reported. Another patient developed a syndrome resembling acute systemic lupus erythematosus, which settled on withdrawal of treatment and flared up on a challenge dose. One patient who experienced facial flushing on chlorpropamide also developed this symptom on tolbutamide. Finally, there was one case of jaundice due to hepatocellular damage, which came on one week after starting treatment.

\section{Phenformin and Metformin}

Sixteen reports were received on reaction to phenformin, the most important being three cases of alcohol-precipitated acute lactic acidosis. There were three cases of rash, one of which was a photodermatitis, two of depression, and two of generalized weakness. There were three reports regarding metformin, with no particular pattern.

\section{Effects on the Fetus}

Five reports were received of effects on the fetus; two describe severe and prolonged hypoglycaemia and convulsions in the newborn. In one case the mother was treated during pregnancy with chlorpropamide and in the other with acetohexamide. Three cases of congenital abnormalities-one of talipes and two with multiple deformities-occurred in babies where the mother had been treated with oral medication. Such deformities are not uncommon and we have no reason to believe that therapy was responsible in any of these cases.

\section{Drug Interactions}

The effect of alcohol in producing flushing in some patients on chlorpropamide is well known. There have been three reports of this; one of these patients also developed flushing while on tolbutamide. Other effects of alcohol, such as the precipitation of an attack of acute paroxysmal tachycardia in one patient on three occasions and in another acute asthma, are not so well known. Alcohol was also responsible for inducing acute lactic acidosis in three patients on phenformin. ${ }^{2} 3$ Another example of drug interaction was severe hypoglycaemia precipitated by phenylbutazone administration in two patients on tolbutamide.

\section{Discussion}

If we assume, and there is some evidence to support this, that the average prescription for insulin or oral medication in diabetes is for two months' supply, it is possible to calculate in a crude manner the number of patients on the various treatment regimens. Excluding those treated on diet alone, it works out that 300,000 patients are on active medication, with about one-third on insulin and the remainder on oral agents. The annual incidence of reported adverse reactions to all oral agents is about one reaction per 15,000 patients and, considering the relatively minor nature of some of the adverse effects, it seems likely that this potent group of drugs has a low degree of acute toxicity. To date on the basis of the "early warning" system data the Committee has not felt the need to take any special action with regard to this group of drugs. No comment can be made regarding possible long-term effects on morbidity or mortality because it is unlikely that such events would have been reported under the present system.

I am grateful to Dr. W. H. W. Inman and Dr. J. F. Dunne for advice in the preparation of this report and to Dr. D. Mansel-Jones for pirmission to publish.

\footnotetext{
References

1 Committee on Safety of Drugs, Annual Report, 1967, London, H.M.S.O. 1968.

Isaac, P., British Medical fournal, 1970, 2, 774.

3 Shirriff,, G. G., and Brewsher, P. D., British Medical fournal, 1970,
} 